\title{
Acute Epiploic Appendagitis: Report of Six Cases and Review of Literature
}

\author{
Antoine El Khoury, Majd Roustom, Henri Azar, Georges Chaer and Tony El Murr* \\ Department of Internal Medicine, Middle East Institute of health, Faculty of Medical Sciences, Lebanese university, Hadat, Lebanon
}

*Corresponding author: Tony El Murr, Department of Internal Medicine, Middle East Institute of health, Faculty of Medical Sciences, Lebanese university, Hadat, Lebanon, Tel: (961)3347473; Email: drtonimurr@hotmail.com

Received: 04 Apr, 2019 | Accepted: 27 Apr, 2019 | Published: 02 May, 2019

Citation: El Khoury A, Roustom M, Azar H, Chaer G, El Murr T (2019) Acute Epiploic Appendagitis: Report of Six Cases and Review of Literature. J Clin Case Stu 4(2): dx.doi.org/10.16966/2471-4925.186

Copyright: (C) 2019 El Khoury A, et al. This is an open-access article distributed under the terms of the Creative Commons Attribution License, which permits unrestricted use, distribution, and reproduction in any medium, provided the original author and source are credited.

\begin{abstract}
Appendagitis has always been considered as a diagnosis of exclusion. Most of the times it is diagnosed incidentally. The majority of patients present flank pain, right and/or left iliac fossa pain with few other symptoms. These findings lead usually the physician toward a surgical disease. Imaging is very important to confirm the diagnosis of appendagitis and to avoid unnecessary hospitalizations and surgeries. In this article, we present five cases of acute appendagitis that have had nearly similar symptoms at presentation and while we were suspecting more severe diseases on their admission at emergency department (ER). The five patients received only medical treatment for their conditions and were discharged home the same day to be reviewed few weeks later and showed complete resolution of the appendagitis on follow up imaging.
\end{abstract}

Keywords: Appendagitis; Appendicitis; Acute abdomen; Thrombosis; Diverticulitis

\section{Introduction}

Appendagitis also known as epiploic appendicitis is a self limiting disease, with benign features that responds to medical treatment in most cases [1]. The central vein that is located in the epiploic appendage can be affected by torsion or thrombosis that will lead to infarction of the epiploic appendage. This condition is 4 times more common in males compared to females and the mean age of diagnosis is $40[2,3]$. The incidence of appendagitis is rare but not well established because in many cases it is falsely considered a diverticulitis or appendicitis [3]. As for the location, it can arise in any colonic segment, most commonly in the rectosigmoid (57\% of the cases) followed by the ileocecum (26\%). The descending colon has the lowest percentage of appendagitis $(2 \%)[4,5]$. Some risk factors increase the prevalence of appendagitis with obesity being the major risk factor because obesity is associated with peritoneal wall thickening and bowel wall thickening. Epiploic appendages are situated on the outer part of colon, the part facing the peritoneum. The formed pouches are filled with fat and are covered by serosa. In each appendage, we find a small artery and vein responsible for the blood supply for a small colonic part. They also contain lymph nodes. The colon contains on average 75 appendages and as mentioned before they are most frequently located in the rectosigmoid colon. The appendages are bigger in size in patients that have excess fat and in those that lost weight very rapidly [6]. They have a protective role during peristalsis. When the appendage is long and large, especially in the case of obese patients, it becomes at risk of torsion that will cause ischemia and infarction of the part supplied by the corresponding vessels. As opposed to acute torsion that produces symptoms, the chronic torsion takes more time to develop and usually is asymptomatic. The most common initial presentation is lower abdominal pain, more on the left but can also occur on the right. The pain is usually constant and does not radiate and can be associated with abdominal distension, nausea, vomiting, diarrhea and low grade fever. These associated symptoms tend to obscure the diagnosis [7]. On physical exam, the patients are hemodynamically stable as they rarely develop fever. The pain is localized to a specific area and does not produce rebound tenderness or peritoneal signs. In very few patients a mass can be detected. Laboratory studies are usually normal and no signs of inflammation [8]. As for the diagnosis, appendagitis is a diagnosis of exclusion. It's found in patients undergoing imaging for other etiologies of lower abdominal pain. Usually it is detected by a CT scan, and when this modality is not available we can use ultrasound but it should not be included in the primary workup $[9,10]$. A CT scan will show a mass that is oval shaped with fat stranding next to it. It may have round shape with fat density and thickened peritoneal lining. [5,10,11]. In addition a central dot that indicates vessel thrombosis $[5,11]$. On ultrasound it appears as a solid, non compressible hyperechoic mass. Doppler studies will show absence of flow [12-14]. The differential diagnosis is very wide and includes all the following: abcess, appendicitis, colon cancer or metastasis, crohn's ilitis, ectopic pregnancy, gallbladder disease, ileitis caused by infection, diverticulitis, mesenteric adenitis, mesenteric panniculitis, ovarian torsion, ruptured or hemorrhagic ovarian cyst, bowel infarction, urachal cyst. The most common being diverticulitis and appendicitis [15]. The treatment is usually medical treatment and is supportive to decrease the duration of symptoms but does not influence the actual disease progression. It consists of non steroidal anti-inflammatory medications for 4 to 6 days and if needed acetaminophen or codeine for 4 to 7 days $[5,16,17]$. Antibiotics are not indicated and most of the patient is discharged home as they do 
not require hospitalization [18]. For patients that fail to improve on medical treatment (progression with high fever, vomiting, diarrhea) or for those that develop complications such as obstruction or abscess require surgery of the affected appendage that should be resected [19].

\section{Case 1: (Adult)}

53 year old man admitted to the ER for subacute left lower quadrant abdominal pain lasting since three weeks, partially relieved by paracetamol. It is mainly an isolated pain and not associated with fever except for the first few days. He weights $95 \mathrm{kgs}$ in his previous medical history, he has only untreated mild hypertriglyceridemia. $\mathrm{He}$ has had an abdominal CT scan with IV contrast that showed a normal urinary tract and a non specific extra-intestinal epiploic inflammatory collection just near the sigmoid colon associated with a few centimetric lymph nodes and mild peritoneal effusion; the aspect was typical of an acute appendagitis; the patient was discharged home on a symptomatic treatment composed by non steroidal anti-inflammatory drug (NSAID) and omeprazole for ten days; at the follow up visit, he was completely asymptomatic and the control abdominal CT scan done three weeks after the diagnosis showed that the collection has totally disappeared.

\section{Case 2: (Adult)}

48 year old obese male patient without a significant medical past history, presented for epigastric pain and left lower abdominal pain. He takes no medications. His symptoms started few days prior to presentation and was associated with nausea and vomiting and undocumented fever. In the ER, he was hemodynamically stable, afebrile. His ECG was normal. He was fully awake and oriented, with normal cardiac and lungs examination. His abdomen was non distended with positive bowel sounds. There was right upper and left lower quadrant abdominal tenderness with a positive Murphy sign. His laboratory workup showed an elevated white count of 14500 with $74 \%$ neutrophils and a normal CRP value. All other findings were normal including liver and pancreatic functional tests. Cardiac enzymes were normal (non-significant). An abdominal ultrasound (abdomen) was initially performed and showed gallbladder wall thickening suggesting an acute cholecystitis. The patient was admitted to the hospital, but his left lower quadrant pain increased in severity. A CT scan of the abdomen and pelvis showed a left colon appendagitis in addition to the gallbladder finding previously detected on ultrasound. The patient has had cholecystectomy followed by three days treatment with NSAID with a complete resolution of his symptoms few days later (Figure 1).

\section{Case 3: (Pediatrics)}

16 years old male with no significant past medical history presented for sudden onset left flank pain radiating to the left inguinal area. He had no urinary symptoms and no other associated complaints. On physical exam he weights $55 \mathrm{kgs}$, his blood pressure was 115/70 and his pulse was 90 beats per minute. His temperature was normal. On physical exam he had a non distended abdomen with normal bowel sounds and mild left sided tenderness. There was no associated costovertebral angle tenderness. His white count was 9000 with $64 \%$ neutrophils and the CRP level was less than 10 . His urine analysis was normal. A CT scan of his abdomen was performed and showed sigmoid appendagitis. He was admitted for 1 day and received NSAIDS and was discharged home on NSAID treatment and his symptoms resolved completely few days later (Figure 2).

\section{Case 4: (Adult)}

22 year old previously healthy female presented for 1 day history

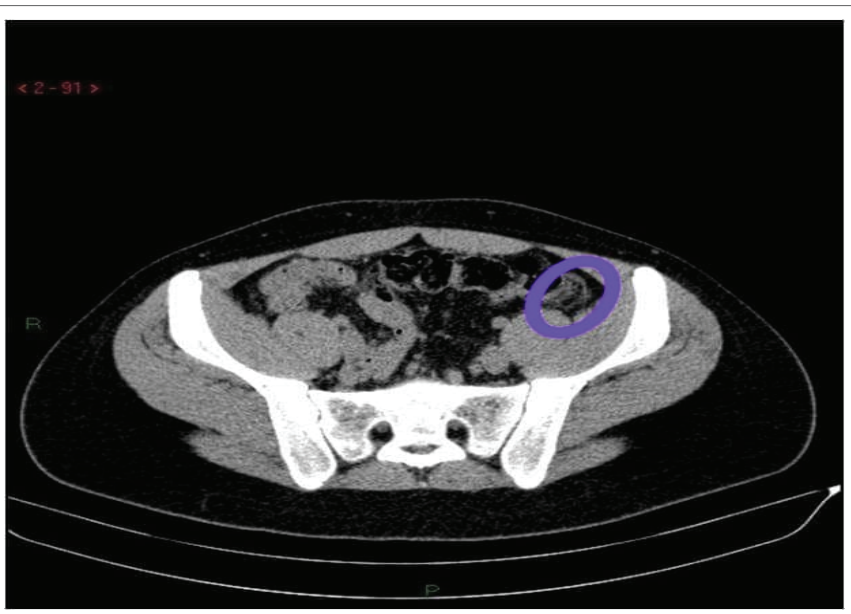

Figure 1: A repeated CT scan of the abdomen showed increase in the size of the appandagitis without associated complications.

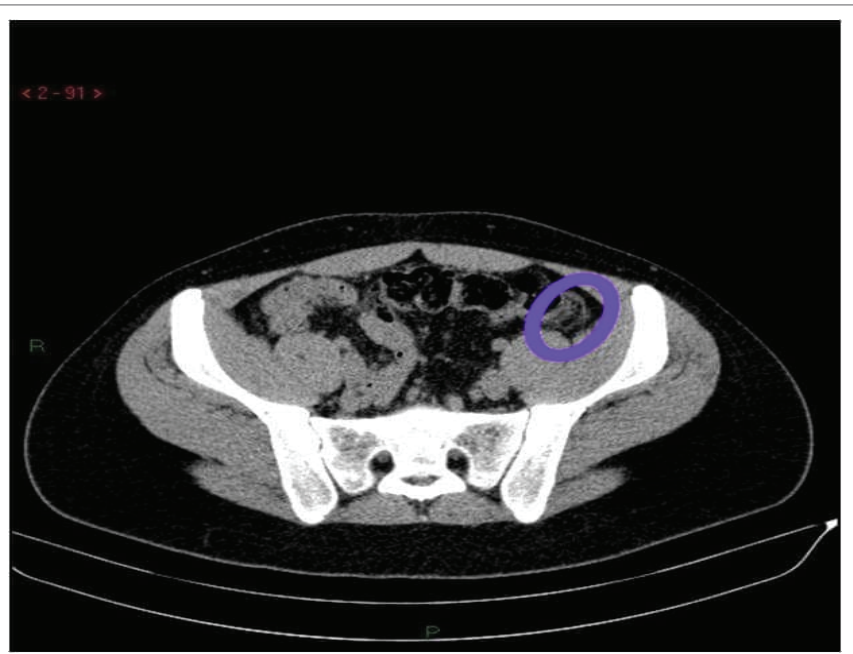

Figure 2: A CT scan of the abdomen showed sigmoid appendagitis.

of left flank pain without urinary symptoms. No vomiting or diarrhea. Her last menstrual period was few days ago. She had normal vital signs and weights $55 \mathrm{kgs}$. Her physical exam showed left flank tenderness. The rest of the examination was normal. Her white count was 7200 with $67 \%$ neutrophils. Her hemoglobin level was 11.1 and the CRP is normal. She had numerous red cells and white cells in her urine analysis so a CT scan was done to rule out pyelonephritis and it showed sigmoid appendagitis only. He was discharged home on medical treatment, and she was symptoms free few days later (Figure 3).

\section{Case 5: (Adult)}

50 year old obese male without significant past medical history presented for right lower quadrant abdominal pain of acute onset. He got admitted due to high suspicion of appendicitis despite normal inflammatory biologic markers. A ct scan showed focal fat infiltration at the level of the peri-cecal region that goes with right iliac fossa appendagitis. There was no evidence of acute appendicitis. The patient was discharged home on medical treatment with complete remission few days later. 


\section{Case 6: (Adult)}

58 year female presented for bilateral flank pain and left lower quadrant pain. She was diagnosed 2 days ago for left sigmoid appendagitis but her pain has increased in intensity and was associated this time with fever. A repeated CT scan of the abdomen showed increase in the size of the appandagitis without associated complications. To mention that the there was infiltrates surrounding both kidneys without signs of pyelonephritis. The patient was continued on non steroidal anti inflammatory medications and her symptoms improved few days later after being admitted to the hospital (Figure 4).

\section{Discussion}

Appendagitis is rare, but if fact it is more common than we think because it's most of the times missed because it requires imaging for diagnosis. Many studies have discussed this point and the two main differential diagnoses are diverticulitis and appendicitis. Our study is in the same direction of other studies and it confirmed the fact that the diagnosis of appendagitis requires the combination of the history and physical exam findings with imaging. We reviewed the files of the 6 patients with appendagitis and compared them with the two other differential diagnoisis. We have found that these patients have symptoms similar to other diseases but most of the times they do not require hospitalization, or require a short course of hospitalization for pain control. They can be discharged and treated at home with a scheduled follow up. Five of our patients have appendagitis located in the left colon while one has it located in the right colon. Only

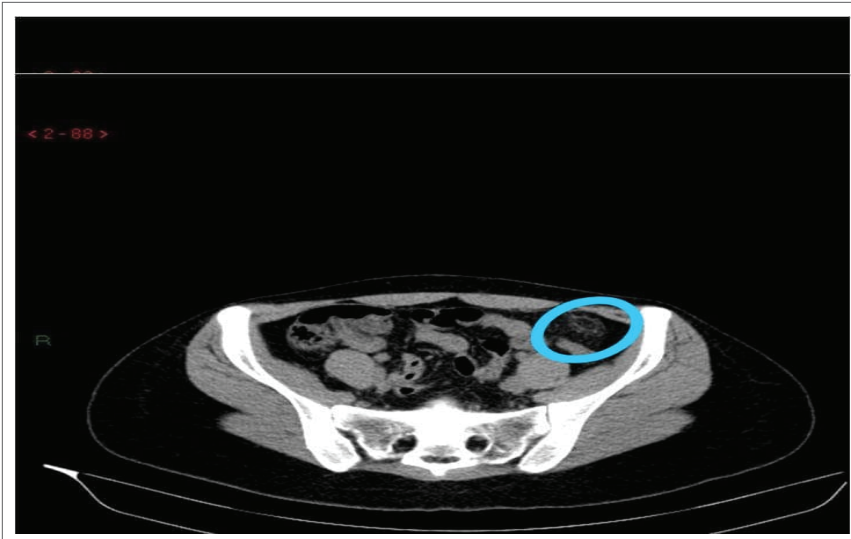

Figure 3: A CT scan of his abdomen showed sigmoid appendagitis.

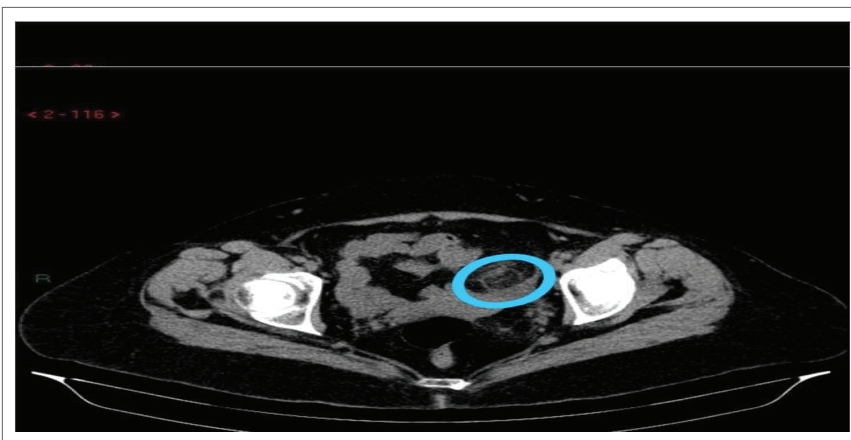

Figure 4: A repeated CT scan of the abdomen showed increase in the size of the appandagitis without associated complications. one patient developed low grade fever with normal inflammatory markers. The white count and CRP was near normal in all cases except for one patient with a white count of 14000 that dropped in follow up labs. Most of our patients are overweight and this is typical for appendagitis and that is compatible with finding in different studies. We would like to mention that any patient, especially overweight patients, presenting with symptoms mimicking acute abdomen and normal inflammatory markers and with no fever or low grade fever, a diagnosis of appendagitis should be suspected and considered as a differential diagnosis.

\section{Conclusion}

Appendagitis is a diagnosis of exclusion. All patients present with symptoms that mimic other diseases and it is important to think about it in our differential diagnosis. The best modality of diagnosis is a CT scan. The 6 cases we presented have all responded to medical treatment consisting of NSAIDS and acetaminophen and they showed complete disease remission on follow up imaging. They did not require antibiotics. Few of them required admission for other reasons.

\section{References}

1. Dockerty MB, Lynn TE, Waugh JM (1956) A clinicopathologic study of the epiploic appendages. Surg Gynecol Obstet 103: 423-433.

2. Schnedl WJ, Krause R, Tafeit E, Tillich M, Lipp RW, et al. (2011) Insights into epiploic appendagitis. Nat Rev Gastroenterol Hepatol 8: 45-49.

3. Ozdemir S, Gulpinar K, Leventoglu S, Uslu HY, Turkoz E, et al. (2010) Torsion of the primary epiploic appendagitis: a case series and review of the literature. Am J Surg 199: 453-458.

4. Macari M, Laks S, Hajdu C, Babb J (2008) Caecal epiploic appendagitis: an unlikely occurrence. Clin Radiol 63: 895-900.

5. Rioux M, Langis $P$ (1994) Primary epiploic appendagitis: clinical, US, and CT findings in 14 cases. Radiology 191: 523-526.

6. Ghahremani GG, White EM, Hoff FL, Gore RM, Miller JW, et al. (1992) Appendices epiploicae of the colon: radiologic and pathologic features. Radiographics 12: 59-77.

7. Sandrasegaran K, Maglinte DD, Rajesh A, Akisik FM (2004) Primary epiploic appendagitis: CT diagnosis. Emerg Radiol 11: 9-14.

8. Carmichael DH, Organ CH Jr (1985) Epiploic disorders. Conditions of the epiploic appendages. Arch Surg 120: 1167-1172.

9. Mollà E, Ripollés T, Martínez MJ, Morote V, Roselló-Sastre E (1998) Primary epiploic appendagitis: US and CT findings. Eur Radiol 8: 435438.

10. Horton KM, Corl FM, Fishman EK (2000) CT evaluation of the colon: inflammatory disease. Radiographics 20: 399-418.

11. Rao PM (1999) CT of diverticulitis and alternative conditions. Semin Ultrasound CT MR 20: 86-93.

12. Deceuninck A, Danse E (2006) Primary epiploic appendagitis: US and CT findings. JBR-BTR 89: 225.

13. Lee YC, Wang HP, Huang SP, Chen YF, Wu MS, et al. (2001) Gray-scale and color Doppler sonographic diagnosis of epiploic appendagitis. J Clin Ultrasound 29: 197-199.

14. van Breda Vriesman AC, Puylaert JB (2002) Epiploic appendagitis and omental infarction: pitfalls and look-alikes. Abdom Imaging 27: 20-28.

15. Garg R, Ma D, Fishbain JT (2018) Epiploic Appendagitis: The Uncommon Intestinal Imitator. Clin Gastroenterol Hepatol 16: A36. 
16. Desai HP, Tripodi J, Gold BM, Burakoff R (1993) Infarction of an epiploic appendage. Review of the literature. J Clin Gastroenterol 16: 323-325.

17. Legome EL, Belton AL, Murray RE, Rao PM, Novelline RA (2002) Epiploic appendagitis: the emergency department presentation. J Emerg Med 22: 9-13.

18. Sangha S, Soto JA, Becker JM, Farraye FA (2004) Primary epiploic appendagitis: an underappreciated diagnosis. A case series and review of the literature. Dig Dis Sci 49: 347-350.

19. Patel VG, Rao A, Williams R, Srinivasan R, Fortson JK, et al. (2007) Cecal epiploic appendagitis: a diagnostic and therapeutic dilemma. Am Surg 73: 828-830. 